\title{
Overview: Wavelet Transform Along With SPIHT Algorithm for Image Compression
}

\author{
Navesh Sallawar ${ }^{1}$, Prof.Nilesh Bodne ${ }^{2}$ \\ Student, Department of Electronics and Communication Engineering, Vidarbha Institute of Technology Nagpur, India ${ }^{1}$ \\ Professor, Department of Electronics and Communication Engineering,Vidarbha Institute of Technology Nagpur,India ${ }^{2}$
}

\begin{abstract}
Image compression is one of the important technologies in multimedia communications, that has been much attention in the past decades, where the two techniques Discrete Wavelet Transform (DWT) and Set-Partitioning In Hierarchical Trees (SPIHT) have great influence on its performance. Due to the properties of fast computation, low memory requirement, adaptive Lifting DWT has been adopted as a new technique for still image compression. Furthermore, the traditional DWT and SPIHT have the drawback of long bits output and time consuming. In this paper we produce a new technique named adaptive Lifting DWT. An Adaptive Lifting DWT that locally adapts the filtering directions to image content based on directional lifting. This technique using the new algorithm that detects all the blocks in a given image to decide whether the block is homogenous or heterogeneous block. For homogeneous block, the simple Discrete Wavelet Transform (DWT) is used. And for the heterogeneous block Lifting Wavelet Transform is used. In this technique image quality is measured objectively, using peak signal noise ratio or picture quality scale, and subjectively, using perceived image quality.
\end{abstract}

Keywords: SPIHT, Entropy coding, Lifting Scheme, Wavelet, Compression.

\section{INTRODUCTION}

Image compression is now essential for applications such as transmission and storage in data bases. Image compression is a technique which is used to compress the data to reduce the storage and transmission time. Image compression is the application of data compression on digital images. The objective is to reduce redundancy of the image data in order to be able to store or transmit data in an efficient form. The compression provides to reduce the cost of storage and increase the speed of transmission. Image compression is used to minimize the size in bytes of a graphics file without degrading the quality of the image. There are two types of image compression is present lossy and loss less[18]. The lossy type aims to reduce the bits required for storing or transmitting an image without considering the image resolution much and the lossless type of image compression focuses on preserving the quality of the compressed image so that it is same as the original image. we use image processing In the coming of era the digitized image is an important challenge to deal with the storage and transmission requirements of enormous data, including medical images. Compression is one of the indispensable techniques to solve this problem. In this paper, we propose an algorithm for medical image compression based on lifting base wavelet transform coupled with SPIHT (Set Partition in Hierarchical Trees) coding algorithm, of which we applied the lifting structure to improve the drawbacks of conventional wavelet transform. We compared the results with various wavelet based compression algorithm. Experimental results show that the proposed algorithm is superior to traditional methods for all tested images at low bit rate. Our algorithm provides better PSNR, Quality factor and MSSIM values for medical images only at low bit rate. Compressed image can be represent in various format such as GIF, JPG, BMP and PNG

\section{WAVELET TRANSFORM}

The wavelet Lifting Scheme is a method for decomposing wavelet transforms into a set of stages. Lifting scheme algorithms have the advantage that they do not require temporary arrays in the calculations steps and have less computation

\section{A. Splitting}

In this stage the input signal is divided in to two disjoint sets, the odd $(X[2 n+1])$ and the even Samples (X [2n]).

\section{B. Lifting}

In this module, the prediction operation $\mathrm{P}$ is used to estimate $\mathrm{X} 0(\mathrm{n})$ from $\mathrm{Xe}(\mathrm{n})$ and results in an Error signal d(n). Then we update $d(n)$ by applying it to the update operation $U$, and the resulting Signal is combined with $X e(n)$ to $S(n)$ estimate, which represents the smooth part of the original Signal. 


\section{Scaling}

A normalization factor is applied to $d(n)$ and $s(n)$, respectively. In the even-indexed part $S(n)$ is Multiplied by a normalization factor Ke to produce the wavelet sub-band XL1. Similarly in the odd index Part the error signals $d$ (n) is multiplied by K0 to obtain the wavelet sub band XH1. The output Result is XL1 and XH1 by using the lifting-based WT are the same as those of using the convolution Approach for the same input. For lifting implementation, the CDF 9/7 wavelet filter pair can be Factorized into a sequence of primal and dual lifting. The most efficient factorization of the poly Phase matrix for the 9/7.SPIHT (Set Partition in Hierarchical Trees) is one of the most advanced schemes, even Outperforming the state-of-the art JPEG 2000 in some situations. The basic principle is the same; a Progressive coding is applied, processing the image respectively to a lowering threshold. The Difference is in the concept of zero trees (spatial orientation trees in SPIHT). There is a coefficient at the highest level of the transform in a particular sub band which considered insignificant against a Particular threshold; it is very probable that its descendants in lower levels will be insignificant too. Therefore we can code quite a large group of coefficients with one symbol. A spatial orientation tree is defined in pyramid constructed with recursive four sub bands splitting. According to this Relationship, the SPIHT algorithm saves many bits that specify insignificant coefficients. The flowchart of SPIHT is presented in Fig. 3 as a First step the original image is decomposed into sub bands. Then the method finds the maximum iteration number. Second, the method puts the DWT Coefficients into a sorting pass that finds the significance coefficients in all coefficients and encodes the sign of these significance coefficients. Third, the significance coefficients that can be found in the sorting pass are put into the refinement pass that uses two bits to exact the reconstruct value for approaching to real value. The first, second and third steps are iterative, and then iteration decreases the threshold (Tn) and the reconstructive value $(\mathrm{Rn}-\mathrm{Rn}-1 / 2)$. As a fourth step, the encoding bits access entropy coding and then transmit or store the bit. The result is in the form of a bit stream. All of the wavelet-basedimage encoding algorithms improve the compression rate and the visual quality, but the wavelet-transform computation is a serious disadvantage of those algorithms.

In the SPIHT technique, though the utilization of the lists is efficient, it is very memory consuming and the passes are not processed simultaneously and hence the processing speed will be relatively low. Hence in order to overcome these problems, Stripe based Parallel SPIHT technique is proposed in this work.

Wavelet coding requires the buffering of the entire image. For medical images, the requirements to store the elements are very high. Hence to overcome this problem, in this paper Stripe buffer is used that stores few lines of wavelet elements that belongs to the same spatial location where only some bits of the image are processed at once instead of entire image. Figure 1 shows embedded wavelet coding based stripe logic and Figure 1 shows the Formation of stripes.

Sorting Pass: It encodes the coefficients that are not significant in higher threshold value and when the threshold value is reduced then it may become significant.

Refinement Pass: In the refinement pass, the nth MSB of the coefficients in the significant pixel list is the final output. Coefficients which are already found to be significant in the previous pass are refined in magnitude by a process of successive Approximation.

\section{III.PERFORMANCE OF IMAGE COMPRESSION SCHEME}

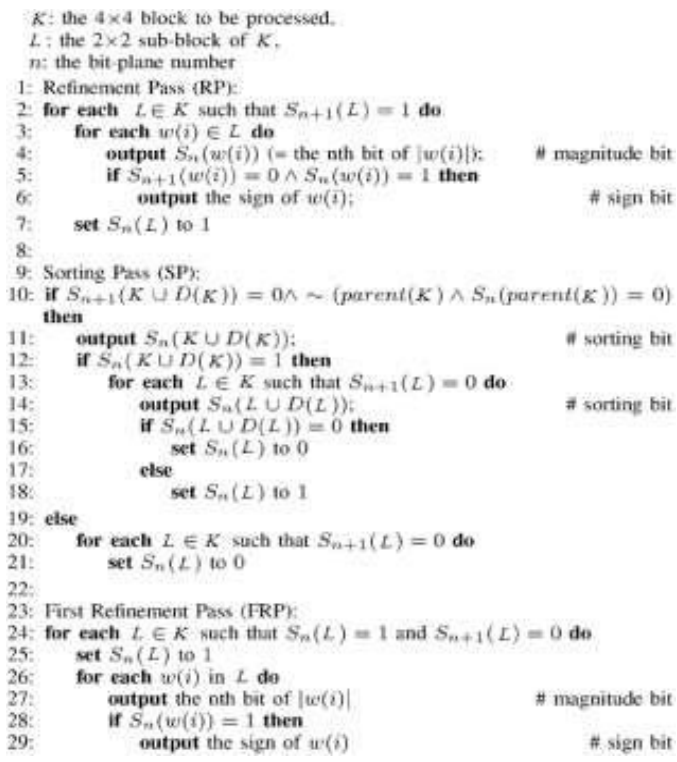


Vol. 8, Issue 4, April 2019

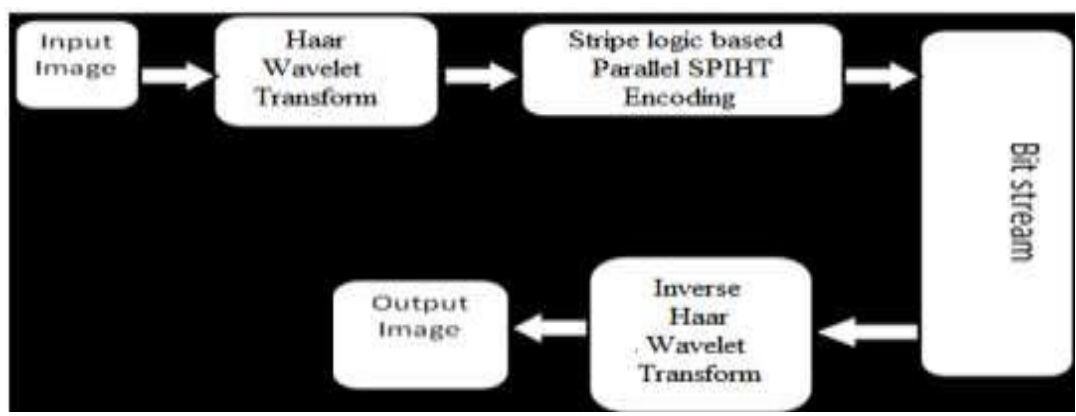

Fig. 1 Block diagram of the entire process

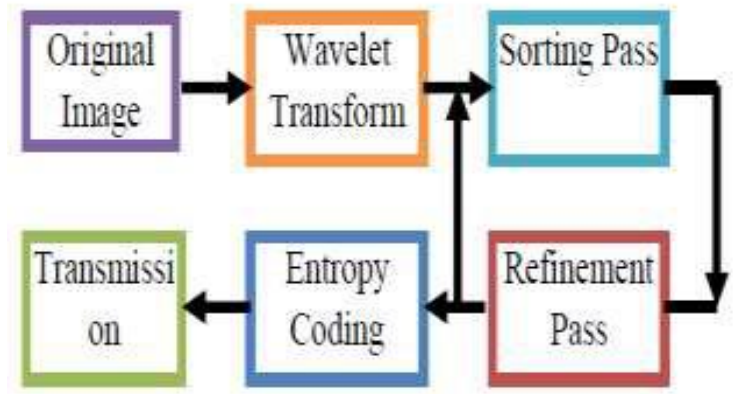

Fig. 2 Flowchart of SPIHT

\section{IV.CONCLUSION}

The proposed method is based on HWT and SPS technique for medical images is presented for real time applications. This technique reduces the memory requirements, enhances the speed and retains all the advantages of embedded coding. The utilization of this method can reduce the MSE or enhance the PSNR values which are the important performance parameters.HWT makes itself a standard technique for its high efficiency. SPIHT which is a powerful technique for still images offers various good characteristics like, good image quality, high PSNR, fast coding and decoding etc., along with HWT technique provides an efficient design. Experimental results demonstrate that the PSNR value obtained by this method may reach up to $83.6 \mathrm{~dB}$. As a future enhancement, the work can be extended for designing higher resolution images of size $512 \times 512,1024 \times 1024$ etc. Number of decomposition levels can be increased that may provide still better compression. Other coding technique can be used instead of bit-plane coding since it is not relevant for decoding to obtain still better results.

\section{REFERENCES}

[1]. Yongseok Jin, Hyuk- Jea Lee, “A Block -based Pass- Parallel SPIHT algorithm”, IEEE, July 2012, PP. 1064-1075.BPS

[2]. Kai Liu, Jie Guo and Evgeniy Belyaev, "VLSI Architecture of Arithmetic coder used in SPIHT", IEEE, Vol 20, April 2012, PP. 697710.neighor

[3]. K. Raghunad Bhattar, K.R, Ramakrishnan and Dasguptha K.S, "Stripe based embedded coding of wavelet coefficients for large images", IISC, 2013. [192]

[4]. B. Priti Bramhankar and B. Sachin, "Lifting based wavelet transform along with SPIHT algorithm used for image compression", IJMTER , July 2013, PP. 2033- 2037. [liftin]

[5]. Yin-hua Wu, Long-xu Jin and Hong-jiang Tao, “An improved fast parallel SPIHT algorithm and its FPGA implementation”, IEEE, 2010, PP. 191-195. [2]

[6]. Youngseop Kim and William A. Pearlman, "Stripe-Based Spiht Lossy Compression of Volumetric Medical Images for Low Memory Usage and Uniform Reconstruction Quality", IEEE, 2000, PP. 652-655

[7]. Mr.Vipin V and Miranda Mathews, "FPGA Implementation of Image Compression using SPIHT technique", IJAREEIE January 2014, PP. 7001-7007.

[8]. Ping Liu, Guanfeng Li, “An Improved SPIHT Algorithm for Image Compression in Low Bit Rate”, Scientific Research, September 2013, PP. 245-248.

\section{BIOGRAPHY}

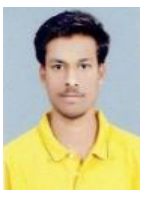

Navesh Sallawar a PG student in Electronics and communication Department, Vidharbha institute of technology Nagpur received BE in Electronics And Telecommunication in 2017 from India. 